\title{
CENPF Upregulation is Associated with Immunosuppressive Status and Predicts Poor Clinical Outcomes in Lung Adenocarcinoma Validated by Qrt- PCR
}

\section{zhiyun xu}

the Affiliated Cancer Hospital of Nanjing Medical University \& Jiangsu Cancer Hospital \& Jiangsu Institute of Cancer Research

\section{Shi Wang}

the Affiliated Cancer Hospital of Nanjing Medical University \& Jiangsu Cancer Hospital \& Jiangsu Institute of Cancer Research

\section{Zhijian Ren}

the Affiliated Cancer Hospital of Nanjing Medical University \& Jiangsu Cancer Hospital \& Jiangsu Institute of Cancer Research

\section{Xiang Gao}

the Affiliated Cancer Hospital of Nanjing Medical University

\section{Lin Xu}

the Affiliated Cancer Hospital of Nanjing Medical University \& Jiangsu Cancer Hospital \& Jiangsu Institute of Cancer Research

\section{Binhui Ren}

the Affiliated Cancer Hospital of Nanjing Medical University \& Jiangsu Cancer Hospital \& Jiangsu Institute of Cancer Research

\section{Shuai Zhang ( $\nabla$ xuezhongdaoke@126.com )}

the Affiliated Cancer Hospital of Nanjing Medical University \& Jiangsu Cancer Hospital \& Jiangsu Institute of Cancer Research

\section{Research Article}

Keywords: CENPF, T cell CD4+ Th2, prognosis, lung adenocarcinoma

Posted Date: December 30th, 2021

DOI: https://doi.org/10.21203/rs.3.rs-1187868/v1

License: (9) (1) This work is licensed under a Creative Commons Attribution 4.0 International License. Read Full License 
Page $2 / 19$ 


\section{Abstract}

Background: CENPF was differentially expressed in various cancers. However, the relationship between CENPF and immune infiltrates in lung adenocarcinoma was previously unknown.

Methods: We implemented a comprehensive analysis of expression of CENPF in the GEO and TCGA databases. CENPF was evaluated for its prognostic value combining clinical samples from the GEPIA2 and TCGA databases. The Metascape together with WebGestalt databases were used for enrichment analysis of genesets that were most postively associated with CENPF. We retrieved the score for immune cell infiltration in TCGA data and examined the correlation between CENPF expression and the infiltration of immune cell by R software.

Results: The outcomes exhibited that up-regulated CENPF mRNA expression was evidently related to poor PFS, DSS and OS in patients with lung adenocarcinoma. Moreover, high expression of CENPF was markedly related to genes associated with immune checkpoint. Further analysis showed that T cell CD4+ Th2 infiltration increased in lung adenocarcinoma samples with high CENPF expression.

Conclusions: Our study indicated that CENPF led to T cell CD4+ Th2 infiltration through oncogenic activity and may be employed as a biomarker for the prediction of prognosis in lung adenocarcinoma.

\section{Introduction}

In the respiratory system, lung adenocarcinoma (LUAD) is one of the most prevalent malignant tumors[1]. It is prone to recurrence and metastasis, as well as the development of treatment resistance, and owns a high mortality $[2,3]$. There are currently no early tumor indicators or diagnostic techniques for LUAD that are enough effective[4]. A growing number of important genes have been identified as a result of the rapid development of transcriptomic research together with high-throughput sequencing techniques $[5,6]$. However, further studies to identify more significant oncogene, particularly those that may influence the configuration of the immune microenvironment in LUAD, are now required.

CENPF encodes a protein that is dynamically expressed throughout the cell cycle[7]. For instance, CENPF has been found to be a latent biomarker for the pancreatic cancer both diagnostically and prognostically[8]. Meanwhile, CENPF serves as a novel biomarker for the nonmuscle invasive bladder cancer that may be utilized to predict disease prognosis and progression, in accordance with the weighted gene co-expression network analysis[9]. Additionally, elevated CENPF expression substantially reduces overall survival in individuals with hepatocellular cancer[10]. Nonetheless, the particular CENPF effect in LUAD is mostly unknown at the moment.

In our research, we investigated at the expression of CENPF in a variety of tumor types from three different cohorts, including TCGA, GTEx, and GEO, and explored its correlation with patient outcomes. The overexpression of CENPF was discovered in LUAD and high expression of CENPF was related to poorer PFS, DSS and OS of LUAD patients. What's more, CENPF was predicted to be implicated in pathways 
related to certain cancer. Since immune cell infiltration affects the prognosis of LUAD patients, we investigated the correlation between the level of immune cell infiltration and CENPF expression and found that T cell CD4+ Th2 infiltration was greater when the expression level of CENPF was high. Our outcomes exhibited that CENPF may exerts a functional effect in LUAD, implying a molecular mechanism by which CENPF affected T cell CD4+ Th2 infiltration in the tumor microenvironment.

\section{Methods And Materials}

\section{Data collection}

The CENPF differential expression in normal samples and 33 cancer was explored using GTEx and TCGA data[11, 12]. All expression profiles are displayed in $\log _{2}(T P M+1)$. The datasets used, including GSE75037, GSE40791 and GSE32863, were from GEO database, and the download data format was MINIML[13, 14]. Additional information on three GSE datasets was included in Table 1.

\section{Table 1}

Details of LUAD studies and associated microarray datasets from GEO database.

\begin{tabular}{|llllll|}
\hline GEO & \multicolumn{2}{l}{ Sample } & & Platform & Country \\
\cline { 2 - 4 } & Tumor & Normal & & USA \\
GSE75037 & 83 & 83 & $\begin{array}{l}\text { GPL6884 Illumina HumanWG-6 v3.0 } \\
\text { expression beadchip }\end{array}$ & USA \\
GSE40791 & 94 & 100 & $\begin{array}{l}\text { GPL570 [HG-U133_Plus_2] Affymetrix Human Genome } \\
\text { U133 Plus 2.0 Array }\end{array}$ & USA \\
\hline GSE32863 & 58 & 58 & $\begin{array}{l}\text { GPL6884 Illumina HumanWG-6 v3.0 expression } \\
\text { beadchip }\end{array}$ & \\
\hline
\end{tabular}

\section{CENPF expression identification}

The mRNA expression of CENPF in LUAD tissues and in corresponding normal lung tissues were explored through the UALCAN database[15]. Moreover, the expression profile of CENPF according to tumor grade was also investigated based on the UALCAN database. The HPA database was utilized for immunohistochemistry (IHC) validation of the CENPF expression level in the LUAD along with normal lung tissues [16].

\section{Cell culture}

The H1299, A549, HBE, H1975 and PC9 cell lines were provided by the Cell Bank of the Chinese Academy of Sciences, which were cultured with $5 \% \mathrm{CO} 2$ under a temperature of $37^{\circ} \mathrm{C}$ in the RPMI- 1640 medium 
(Gibco) added with penicillin/streptomycin (1\%, Gibco) and fetal bovine serum (10\%, Biological Industries).

\section{qRT-PCR}

TRIzol reagent (Invitrogen, USA) was utilized for extracting the total RNA respectively from H1299, A549, H1975 and PC9 cells. The first-strand cDNA could be generated from the total RNA with reverse transcriptase and oligo-dT primers (Invitrogen, USA). QuantiTect SYBR Green PCR Master Mix (Qiagen, Germany) was employed to conduct qRT-PCR. On the basis of CENPF mRNA sequence, primers could be designed in GenBank. Additional information regarding primer sequences was available in Table 2. As an endogenous control, $\beta$-actin was identified in each experimental sample. All responses were performed three times.

Table 2

The sequences information of qRT-PCR primers

\begin{tabular}{|lll|}
\hline Gene name & Primers (5'-3') & \\
\hline CENPF & Forward: CTCTCCCGTCAACAGCGTTC & Reverse: GTTGTGCATATTCTTGGCTTGC \\
\hline B-actin & Forward: CTCCATCCTGGCCTCGCTGT & Reverse: GCTGTCACCTTCACCGTTCC \\
\hline
\end{tabular}

\section{Survival analysis}

With the aim of investigating the latent prognostic effect of CENPF in TCGA pan-cancer, the associations between the expression of CENPF and OS in patients with LUAD were implemented via GEPIA2 database[17]. From TCGA, the clinical data of 33 cancer patients could be downloaded, univariate cox regression was applied to analyze PFS and DSS. The R software package "forestplot" was employed for visualizing $95 \%$ confidence interval, $\mathrm{HR}$, and $\mathrm{p}$ value of each variable utilizing a forest map.

\section{Correlation and enrichment analysis}

To conduct enrichment analysis, the top 300 genes with the positively pearson correlation coefficient in LUAD from TCGA data were selected to reveal the function of CENPF. Then, the Ontology (GO) and genome encyclopedia (KEGG) analyses of the above gene sets were performed using Metascape[18]. Meanwhile, utilizing WebGestalt, Gene set enrichment analysis (GSEA) was implemented in order to further investigate CENPF function[19, 20].

\section{Immune cell infiltration analysis}


We divided LUAD samples from TCGA into two groups in accordance with the median expression level s(high CENPF and low CENPF) for assessing the level of immune cell infiltration in LUAD tissues. Then, with $x$ Cell algorithm, the immune cells infiltration were estimated according to $R$ package "Immuneeconv". CENPF subgroups were represented on the horizontal axis of heat maps, distinct immune scores were displayed on the vertical axis, and correlation coefficients were shown in various colors. In addition, utilizing TIMER2.0 database, the association between the expression profile of CENPF and the proportion of immune infiltration of T cell CD+ Th2 in LUAD was confirmed[21].

\section{Correlation between CENPF expression and immune checkpoint-related genes, TMB or MSI}

The relationship between genes related to immune checkpoint and CENPF were studied using LUAD mRNA sequencing data from TCGA. Furthermore, the association between the expression of CENPF and MSI or TMB in LUAD were analyzed using the R package "ggstatsplot" based on TCGA data, respectively. All the above analysis results were displayed by R packages "ggplot2" and "pheatmap".

\section{Statistical analysis}

All of the data were performed with $\mathrm{R}$ version 4.0.3. The levels of CENPF that were differentially expressed between malignant and non-malignant lung samples were estimated by the Student t-test. The relationships between the immune cells infiltration level and CENPF were investigated through exploiting spearman's correlation. The Spearman's correlation was exploited to express the relationships between the immune cells infiltration level and CENPF. Then $p<0.05$ were regarded statistically significant.

\section{Results}

\section{Pan-cancer CENPF expression analysis}

CENPF expression in the pan-cancer data from GTEx and TCGA was detected initially. The analysis exhibited the expression of CENPF was revealed to be elevated in 29 kinds of cancer, containing BRCA, BLCA, ACC, COAD, CHOL, CESC, GBM, ECSA, DLBC, KIRC, KICH, HNSC, LIHC, LGG, KIRP, OV, LUSC, LUAD, PRAD, PCPG, PAAD, SKCM, SARC, STAD, READ, THCA, TGCT, UCS and UCEC (Figure 1A-1D). In addition, CENPF was highly expressed in LUAD based on GSE75037, GSE32863 and GSE40791 (Figure $1 \mathrm{E}-1 \mathrm{G})$. The analysis of UALCAN database showed that the expression of CENPF was up-regulated in the tumor tissues of LUAD patients as in contrast to the adjacent normal tissues (Figure 2A). Simultaneously, the CENPF expression level in LUAD increased with the progression of tumor grade (Figure 2B). Moreover, IHC outcomes in the HPA database exhibited that the expression of CENPF was evidently up-regulated in LUAD lungs in contrast to controls containing normal histology (Figure 2C). The CENPF expression was 


\section{Association between CENPF expression and cancer patient prognosis}

We explored the relationship between the expression of CENPF and PFS, DSS, OS and in different kinds of tumors utilizing GEPIA2 database and the TCGA cohort for determining CENPF value in the prediction of cancer patient prognosis. The outcomes indicated higher expression of CENPF was related to an evident decreased OS in LGG $(p=1.8 \mathrm{e}-05), \operatorname{KIRC}(p=0.0036), \operatorname{ACC}(p=0.00024)$, LUAD (with $p$ value of $0.0098), \operatorname{LIHC}(p=0.0018), \operatorname{KIRP}$ (with $p$ value of 0.00046$)$, MESO $(p=1.8 \mathrm{e}-07), \operatorname{SARC}(p=0.012), \operatorname{SKCM}$ $(p=0.024$; Figure 3A-3I). However, THKM $(p=0.0074)$ demonstrated a tendency toward better survival as CENPF expression increased (Figure $3 \mathrm{~J})$. Besides, Increased CENPF expression was correlated to a reduced DSS in $\operatorname{KIRP}(p=3 \mathrm{e}-04), \operatorname{KIRC}(p=1 \mathrm{e}-04), \operatorname{ACC}(p<0.0001), \operatorname{LUAD}(p<0.0013), \operatorname{LIHC}(p=$ $0.0063), \operatorname{LGG}(p<0.0001), \operatorname{SARC}(p=0.0403), \operatorname{PAAD}(p=0.0287), \operatorname{MESO}(p<0.0001)$, $\operatorname{SKCM}(p=0.0023$; Supplementary Figure 1). With higher CENPF expression, PFS decreased in the ACC ( $p=2 \mathrm{e}-04), \mathrm{GBM}(p=$ $7 \mathrm{e}-04), \operatorname{CESC}(p=0.0367), \operatorname{BLCA}(p=0.0405), \operatorname{LGG}(p=0.0012), \operatorname{KIRP}(p<0.0001), \operatorname{KIRC}(p=3 \mathrm{e}-04)$, MESO $(p=0.0021), \operatorname{LUAD}(p=0.025), \operatorname{LIHC}(p=9 \mathrm{e}-04), \operatorname{THCA}(p=0.0162), \operatorname{SARC}(p=0.0048), \operatorname{PRAD}(p=$ $0.0024), \operatorname{UCEC}(p=0.0265)$ and UVM $(p=0.0036$; Supplementary Figure 2$)$.

\section{Correlation and enrichment analysis}

To ascertain the role of CENPF, we utilized TCGA data to conduct a correlation study between CENPF and other LUAD genes. For enrichment analysis, the first 300 genes remarkably associated with CENPF were chose (Supplementary Table 1). We next used Metascape to thoroughly investigate biological pathways with above 300 genes. Gene Ontology (GO) analysis revealed that mitotic cell cycle process, cell cycle process regulation together with microtubule skeleton organization were significantly enriched in BP; microtubule binding, ATP-dependent activity and chromatin binding were markedly abundant in MF; spindle, spindle pole, as well as chromosomal region were significantly enriched in CC (Figure 4A-4C). Moreover, the study of KEGG pathway suggested that DNA replication, fanconi anemia pathway and cell cycle were obviously abundant (Figure 4D). GSEA was employed to determine Reactome and KEGG pathways, which revealed that the progesterone-mediated oocyte maturation and the degradation of mitotic proteins pathways mediated by APC/C and APC/C:Cdc20 activation were significantly enriched (Figure 4E-4F). These findings showed that CENPF was participated in various pathways related to cancer in LUAD.

\section{CENPF expression and immune cell infiltration analysis}


Further investigation of LUAD immune cell infiltration score in TCGA revealed that the level of T cell CD4+ Th2 infiltration was substantially higher in the group with high CENPF expression (Figure 5A-5B). This result indicated that high expression CENPF promoted the infiltration level of T cell CD4+ Th2, which demonstrated a correlation with the immunosuppressive state in LUAD[22]. T cell CD4+ Th2 expression was significantly enhanced in tumor patients, which promoted the occurrence and development of malignancies by decreasing cellular immune function[23]. Likewise, we discovered that three indicators scored substantially lower on groups of high CENPF expression, including immune score, microenvironment score, and stroma score (Figure 5C-5E). As well, we further verified the significantly positive correlation between the T cell CD 4 + Th2 infiltration level and CENPF expression in the LUAD tissues through Timer 2.0 database (Figure 6A).

\section{Correlation between CENPF and immune checkpoint- related genes, TMB or MSI in LUAD}

Subsequently, the significantly positive correlation between TMB/MSI score and the CENPF expression level in LUAD was found (Figure 6B-6C). Then, we analyzed the correlation between CENPF and immune checkpoint-related genes using LUAD data in TCGA and found that CENPF was also highly positively correlated with immune checkpoint-related genes in LUAD, such as CD274, KIR2DL3, LAG3, LARS1, NECTIN2, PDCD1, TGFBR1 and TIGIT (Figure 6D). Following these findings, it was hypothesized that increased CENPF expression was strongly associated with immunosuppressive state in patients with LUAD[24]. Moreover, several immune checkpoints were chosen with the aim of exploring differential expression levels between high CENPF expression and low CENPF expression, and found that CD274, LAG3, PDCD1 and TIGIT were substantially increased in the group with high CENPF expression (Figure $6 \mathrm{E})$. These outcomes exhibited that high expression of CENPF was associated with tumor immunosuppression in LUAD patients. The results presented CENPF was positively correlated with many known immune checkpoints so as to inhibit the immune state of tumor. However, the score of TMB/MSI indicated that there may be potential immune checkpoints to be found and the immunosuppression status of LUAD could be effectively improved in patients with LUAD containing high CENPF expression.

\section{Discussion}

Although CENPF was identified in the development of many malignancies, it had not been widely studied in various types of cancer[25,26]. Hence, the effect of CENPF in the cancer progression and prognosis must be clarified as rapidly as possible. CENPF has been involved in a variety of cancers, according to previous research. For instance, CENPF can stimulate the metastasis of breast cancer bone through triggering PI3K-AKT-mTORC1 signaling, suggesting it as a new target for breast cancer therapy[27]. Meanwhile, Ping-An Zou et al. found that higher CENPF levels led to osteosarcoma cell proliferation through regulating apoptosis and the cell cycle[28]. Based on current study, CENPF boosted the progression of papillary thyroid carcinoma through controlling cell proliferation and apoptosis[29]. 
According to our findings, CENPF was significantly expressed in COAD, CHOL, BRCA, LAML, GBM, ESCA, LUAD, LIHC, LGG, STAD, PAAD, OV, UCS and TGCT, while it was expressed at a low level in HNSC, KICH, KIRC, KIRP, READ, SKCM, and THCA according to TCGA and GTEx data. Additionally, the GSE75037, GSE40791, and GSE32863 datasets revealed that CENPF was significantly expressed in LUAD. There could be a discernible difference in CENPF expression across tumor types, which might also suggest different underlying actions and mechanisms. Furthermore, we discovered that upregulation CENPF was related to worse prognosis in individuals with tumors expressing a high level of CENPF. However, its expression was related to poor prognosis in THKM. These findings revealed CENPF might be a beneficial biomarker for the prediction of the cancer patients prognosis.

Tumor microenvironment immune cells were a critical component of tumor tissues, with mounting data demonstrating their clinicopathological role in predicting tumor individual survival and treatment effectiveness[30,31]. We also discovered that when the connection between CENPF and immune cell infiltration was analyzed, the amount of T cell CD4+ Th2 infiltration was substantially greater in the group containing high expression of CENPF. CENPF also revealed a remarkable correlation with genes involved in immune checkpoint-related genes, indicating a function in tumor immunology regulation. Specifically, the infiltration level of T cell Cd4+ Th2 promotes the progression of LUAD with high CENPF.

\section{Conclusion}

In summary, CENPF is likely to be involved in in the infiltration of immune cell and may be a useful predictive biomarker for lung adenocarcinoma.

\section{Abbreviations}

TCGA: The Cancer Genome Atlas; GEO: Gene Expression Omnibus; IHC: Immunohistochemistry; GO: Gene Ontology; KEGG: Kyoto Encyclopedia of Genes and Genomes; GSEA: Gene Set Enrichment Analysis; TMB: Tumor mutational burden MSI: Microsatellite instability; OS: Overall survival; DSS: Disease-specific survival; PFS: Progression-free survival; LUAD: Lung adenocarcinoma; ACC: Adrenocortical carcinoma; BLCA: Bladder urothelial carcinoma; BRCA: Breast invasive carcinoma; CESC: Cervical squamous cell carcinoma and endocervical adenocarcinoma; CHOL: Cholangiocarcinoma; COAD: Colon adenocarcinoma; DLBC: Lymphoid neoplasm diffuse large B-cell lymphoma; ECSA: Esophageal carcinoma; GBM: Glioblastoma multiforme; HNSC: Head and neck squamous cell carcinoma; $\mathrm{KICH}$ : Kidney chromophobe; KIRC: Kidney renal clear cell carcinoma; KIRP: Kidney renal papillary cell carcinoma; LGG: Brain Lower Grade Glioma; LIHC: Liver hepatocellular carcinoma; LUSC: Lung squamous cell carcinoma; OV: Ovarian serous cystadenocarcinoma; PAAD: Pancreatic adenocarcinoma; PCPG: Pheochromocytoma and paraganglioma; PRAD: Prostate adenocarcinoma; READ: Rectum adenocarcinoma; SARC: Sarcoma; SKCM: Skin cutaneous melanoma; STAD: Stomach adenocarcinoma; TGCT: Testicular germ cell tumors; THCA: Thyroid carcinoma; UCEC: Uterine corpus endometrial carcinoma; UCS: Uterine carcinosarcoma 


\section{Declarations}

\section{Acknowledgements}

We would like to express our gratitude to the team members for their contributions to this article, and then we will work diligently to do relevant research in the future.

\section{Authors' contributions}

Binhui Ren, Shuai Zhang, and Zhiyun Xu participated in the whole study design. The manuscript was drafted by Zhiyun Xu, Shi Wang and Zhijian Ren, revised by Zhiyun Xu, Xiang Gao, and Lin Xu. The authors read and approved the final manuscript.

\section{Funding}

This study was supported by the grants from the Nation Natural Science Foundation of China (81903992), Youth Foundation of Jiangsu Commission of Health (No. Q2017004), Jiangsu Provincial Medical Youth Talent (No. QNRC2016656) and Six talent peaks project in Jiangsu Province (No.WSN042).

\section{Availability of data and materials}

The datasets used and/or analyzed during the current study are available from the corresponding author on reasonable request.

\section{References}

1. Liang M, Chen M, Zhong Y, Singh S, Singh S: Construction of a Prognostic Model in Lung Adenocarcinoma Based on Ferroptosis-Related Genes. Front Genet2021, 12:739520.

2. Wei C, Dong X, Lu H, Tong F, Chen L, Zhang R, Dong J, Hu Y, Wu G, Dong X: LPCAT1 promotes brain metastasis of lung adenocarcinoma by up-regulating PI3K/AKT/MYC pathway. J Exp Clin Cancer Res2019, 38(1):95.

3. Lung Cancer Incidence and Mortality with Extended Follow-up in the National Lung Screening Trial. J Thorac Onco/2019, 14(10):1732-1742.

4. Zengin T, Önal-Süzek T: Analysis of genomic and transcriptomic variations as prognostic signature for lung adenocarcinoma. BMC Bioinformatics2020, 21(Suppl 14):368.

5. Reuter JA, Spacek DV, Snyder MP: High-throughput sequencing technologies. Mol Cel/2015, 58(4):586-597.

6. Pan-cancer analysis of whole genomes. Nature2020, 578(7793):82-93. 
7. Li M-X, Zhang M-Y, Dong H-H, Li A-J, Teng H-F, Liu A-L, Xu N, Qu Y-Q: Overexpression of CENPF is associated with progression and poor prognosis of lung adenocarcinoma. Int J Med Sci2021, 18(2):494-504.

8. Cheng Y, Wang K, Geng L, Sun J, Xu W, Liu D, Gong S, Zhu Y: Identification of candidate diagnostic and prognostic biomarkers for pancreatic carcinoma. EBioMedicine2019, 40:382-393.

9. Shi J, Zhang P, Liu L, Min X, Xiao Y: Weighted gene coexpression network analysis identifies a new biomarker of CENPF for prediction disease prognosis and progression in nonmuscle invasive bladder cancer. Mol Genet Genomic Med2019, 7(11):e982.

10. Huang $Y$, Chen X, Wang L, Wang T, Tang X, Su X: Centromere Protein F 0 Serves as a Potential Prognostic Biomarker and Target for Human Hepatocellular Carcinoma. J Cancer2021, 12(10):29332951.

11. The Genotype-Tissue Expression (GTEx) project. Nat Genet2013, 45(6):580-585.

12. Blum A, Wang P, Zenklusen JC: SnapShot: TCGA-Analyzed Tumors. Cel/2018, 173(2):530.

13. Barrett T, Wilhite SE, Ledoux P, Evangelista C, Kim IF, Tomashevsky M, Marshall KA, Phillippy KH, Sherman PM, Holko Met al: NCBI GEO: archive for functional genomics data sets-update. Nucleic Acids Res2013, 41(Database issue):D991-D995.

14. Chen G, Ramírez JC, Deng N, Qiu X, Wu C, Zheng WJ, Wu H: Restructured GEO: restructuring Gene Expression Omnibus metadata for genome dynamics analysis. Database (Oxford)2019, 2019.

15. Chandrashekar DS, Bashel B, Balasubramanya SAH, Creighton CJ, Ponce-Rodriguez I, Chakravarthi BVSK, Varambally S: UALCAN: A Portal for Facilitating Tumor Subgroup Gene Expression and Survival Analyses. Neoplasia2017, 19(8):649-658.

16. Navani S: Manual evaluation of tissue microarrays in a high-throughput research project: The contribution of Indian surgical pathology to the Human Protein Atlas (HPA) project. Proteomics2016, 16(8):1266-1270.

17. Tang Z, Kang B, Li C, Chen T, Zhang Z: GEPIA2: an enhanced web server for large-scale expression profiling and interactive analysis. Nucleic Acids Res2019, 47(W1):W556-W560.

18. Zhou Y, Zhou B, Pache L, Chang M, Khodabakhshi AH, Tanaseichuk O, Benner C, Chanda SK: Metascape provides a biologist-oriented resource for the analysis of systems-level datasets. Nat Commun2019, 10(1):1523.

19. Liao Y, Wang J, Jaehnig EJ, Shi Z, Zhang B: WebGestalt 2019: gene set analysis toolkit with revamped Uls and APIs. Nucleic Acids Res2019, 47(W1):W199-W205.

20. Wang J, Vasaikar S, Shi Z, Greer M, Zhang B: WebGestalt 2017: a more comprehensive, powerful, flexible and interactive gene set enrichment analysis toolkit. Nucleic Acids Res2017, 45(W1):W130W137.

21. Li T, Fu J, Zeng Z, Cohen D, Li J, Chen Q, Li B, Liu XS: TIMER2.0 for analysis of tumor-infiltrating immune cells. Nucleic Acids Res2020, 48(W1):W509-W514. 
22. Levine AG, Mendoza A, Hemmers S, Moltedo B, Niec RE, Schizas M, Hoyos BE, Putintseva EV, Chaudhry A, Dikiy Set al: Stability and function of regulatory T cells expressing the transcription factor T-bet. Nature2017, 546(7658):421-425.

23. Lee WS, Yang H, Chon HJ, Kim C: Combination of anti-angiogenic therapy and immune checkpoint blockade normalizes vascular-immune crosstalk to potentiate cancer immunity. Exp Mol Med2020, 52(9):1475-1485.

24. Tian M, Yang J, Han J, He J, Liao W: A novel immune checkpoint-related seven-gene signature for predicting prognosis and immunotherapy response in melanoma. Int Immunopharmaco/2020, 87:106821.

25. Aytes A, Mitrofanova A, Lefebvre C, Alvarez MJ, Castillo-Martin M, Zheng T, Eastham JA, Gopalan A, Pienta KJ, Shen MMet al: Cross-species regulatory network analysis identifies a synergistic interaction between FOXM1 and CENPF that drives prostate cancer malignancy. Cancer Cel/2014, 25(5):638-651.

26. Chen X, Zhang D, Jiang F, Shen Y, Li X, Hu X, Wei P, Shen X: Prognostic Prediction Using a Stemness Index-Related Signature in a Cohort of Gastric Cancer. Front Mol Biosci2020, 7:570702.

27. Sun J, Huang J, Lan J, Zhou K, Gao Y, Song Z, Deng Y, Liu L, Dong Y, Liu X: Overexpression of CENPF correlates with poor prognosis and tumor bone metastasis in breast cancer. Cancer Cell Int2019, 19:264.

28. Zou P-A, Yang Z-X, Wang X, Tao Z-W: Upregulation of CENPF is linked to aggressive features of osteosarcoma. Oncol Lett2021, 22(3):648.

29. Han Y, Xu S, Cheng K, Diao C, Liu S, Zou W, Bi Y: CENPF promotes papillary thyroid cancer progression by mediating cell proliferation and apoptosis. Exp Ther Med2021, 21(4):401.

30. Sathe A, Grimes SM, Lau BT, Chen J, Suarez C, Huang RJ, Poultsides G, Ji HP: Single-Cell Genomic Characterization Reveals the Cellular Reprogramming of the Gastric Tumor Microenvironment. Clin Cancer Res2020, 26(11):2640-2653.

31. Ge P, Wang W, Li L, Zhang G, Gao Z, Tang Z, Dang X, Wu Y: Profiles of immune cell infiltration and immune-related genes in the tumor microenvironment of colorectal cancer. Biomed Pharmacother2019, 118:109228.

\section{Figures}



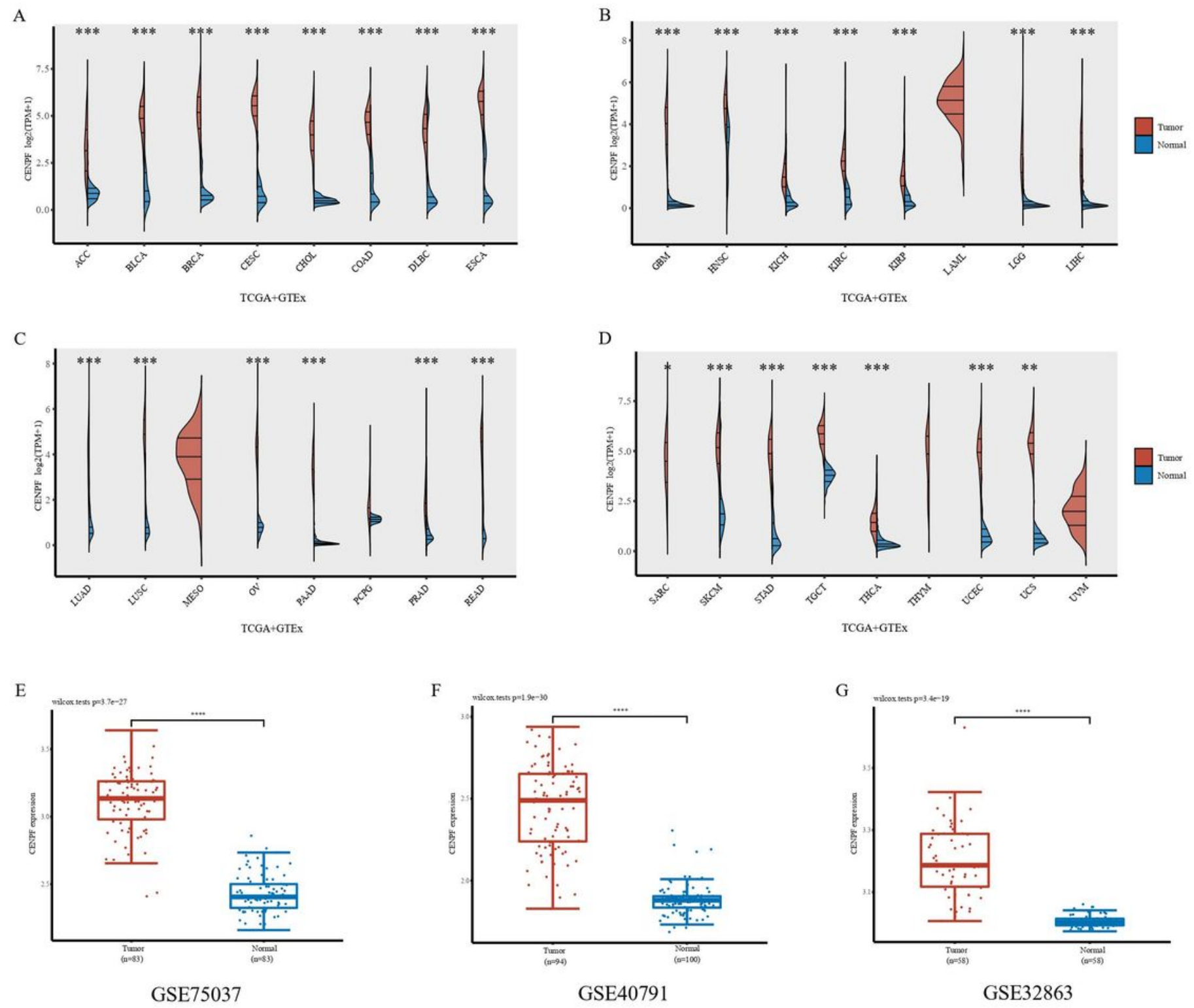

Figure 1

Pan-cancer CENPF expression analysis. (A-D) CENPF expression in tumor together with normal tissues in TCGA and GTEx pan-cancer data. (E-G) CENPF expression in tumor and normal tissues in LUAD from GSE75037, GSE40791 and GSE32863, respectively. $* p<0.05 ; * \star p<0.01 ; * \star * p<0.001$. 
A

Expression of CENPF in LUAD based on Sample types

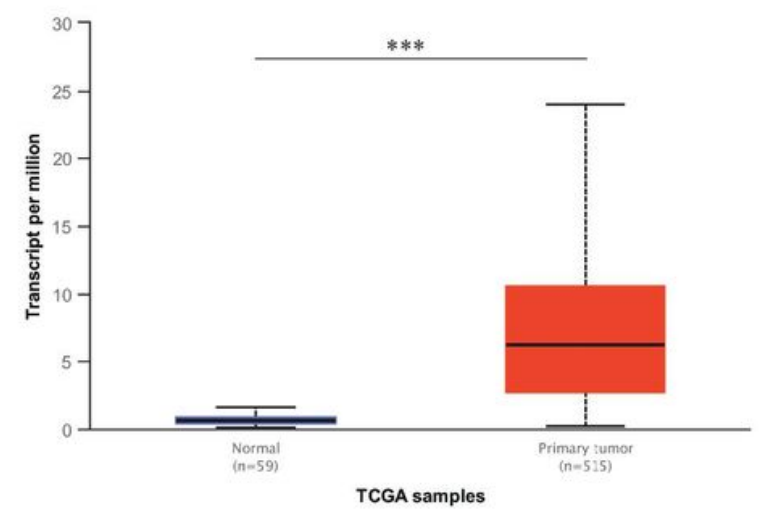

C
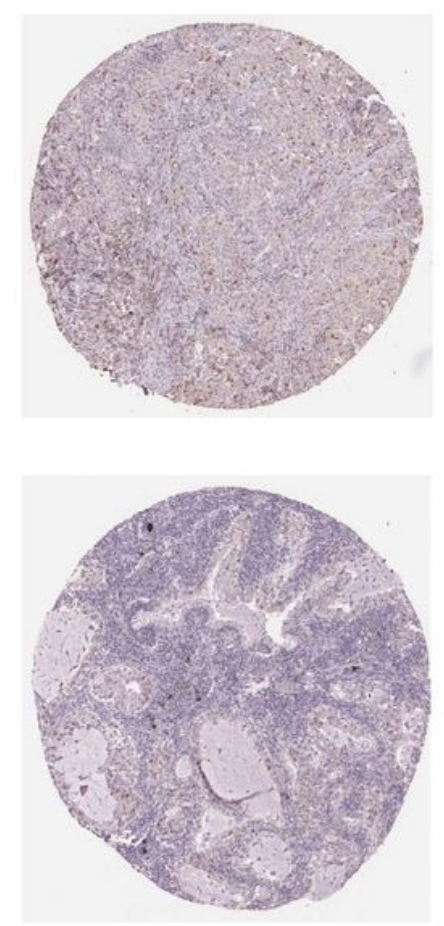

Patient id: 3144

Age: 73

Staining: Medium Intensity: Moderate Quantity: $>75 \%$
B

Expression of CENPF in LUAD based on individual cancer stages

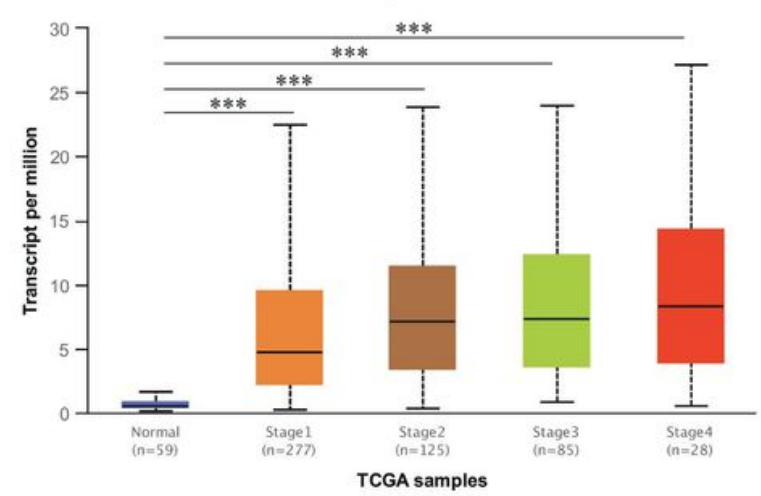

D

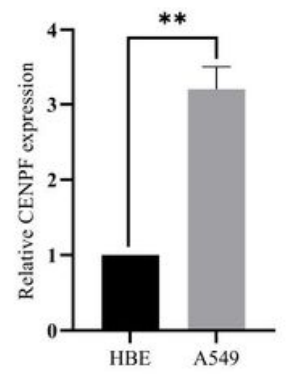

E

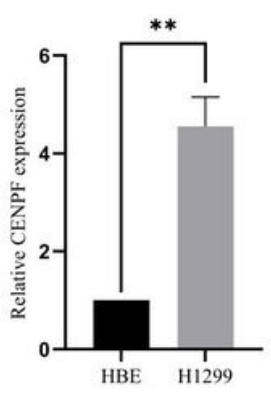

F

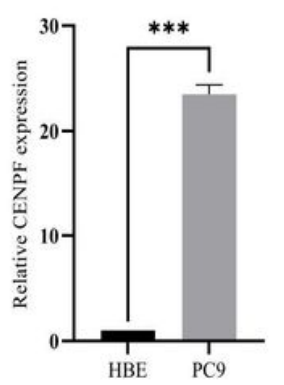

G

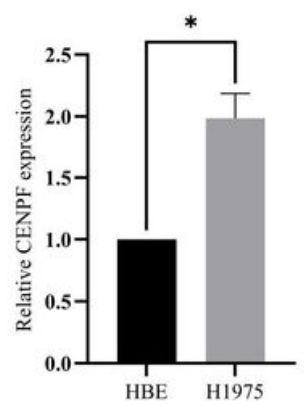

Figure 2

Expression of CENPF in LUAD. (A) Results from public database UALCAN indicated CENPF expression in LUAD is higher than adjacent non-tumor lung tissues. (B) CENPF mRNA expression was remarkably positively associated with pathological stage in LUAD. (C) The Human Protein Atlas database indicates that CENPF is strongly expressed in LUAD. (D-G) Quantitative real-time PCR (qRT-PCR) analysis indicated that CENPF expression in A549, H1299 and PC9 cells was higher compared to HBE cells. ${ }^{*} p<0.05$; $* \star p<$ $0.01 ; * \star \star p<0.001$. 

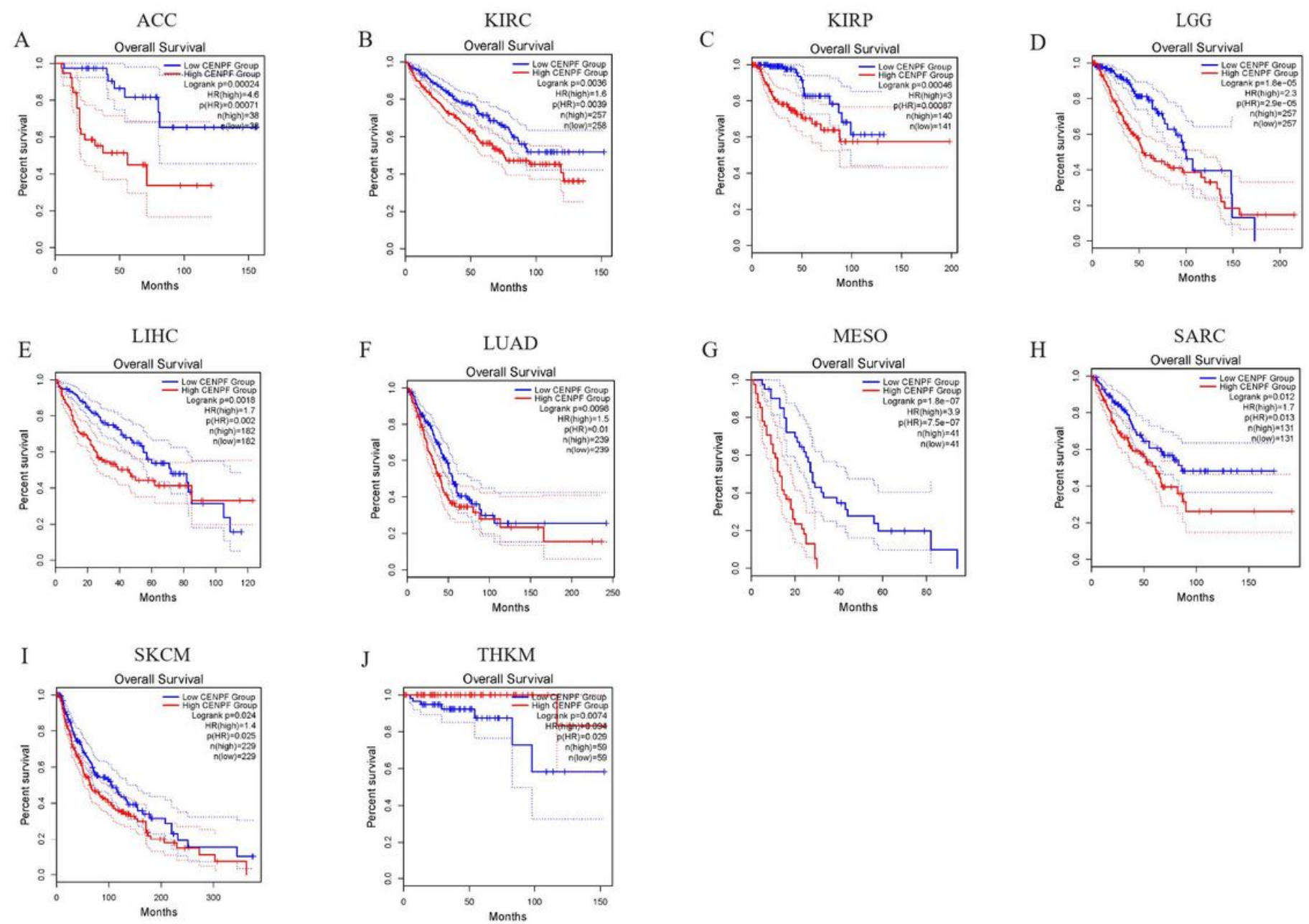

\section{Figure 3}

Association between CENPF expression and pan-cancer prognosis. (A-I) GEPIA2 analysis of OS showed that higher CENPF expression indicated a poorer prognosis of pan-cancer, including ACC, BLCA, BRCA, CESC, CHOL, COAD, DLBC, ECSA, KICH, HNSC, GBM, LGG, KIRP, KIRC, LUSC, LUAD, LIHC, PCPG, PAAD, OV, SARC, READ, PRAD, TGCT, STAD, SKCM, UCS, UCEC and THCA. (J) GEPIA2 analysis of OS indicated that lower CENPF expression showed a poorer prognosis in THKM. Results with logrank $p<0.05$ were shown. 
A

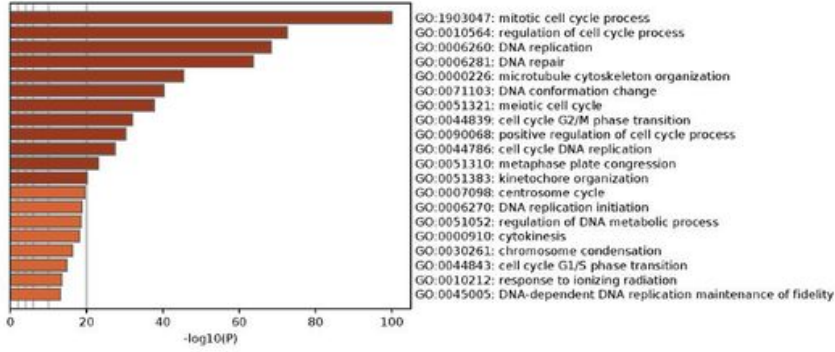

C

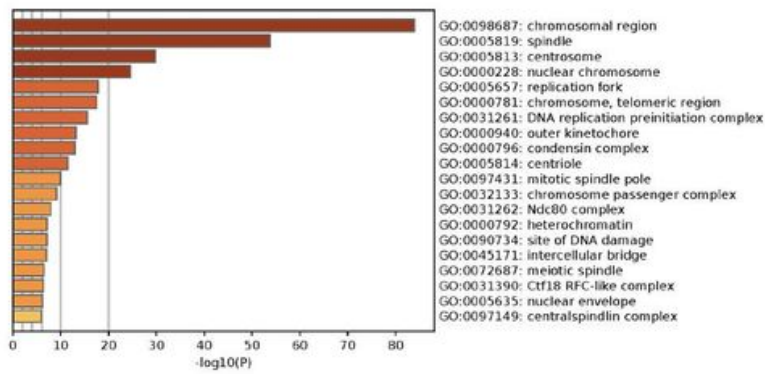

E

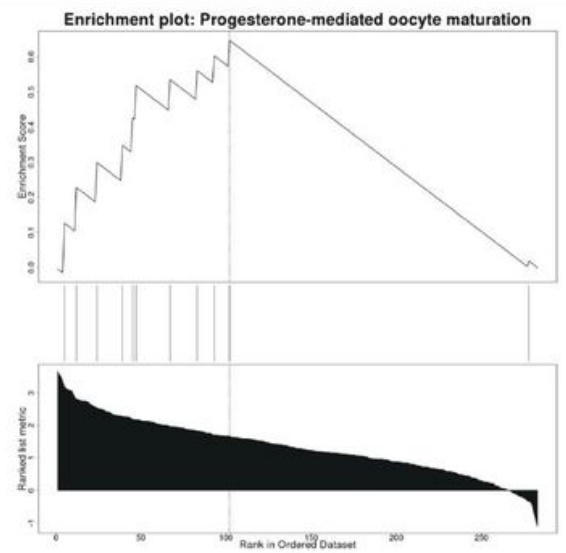

B

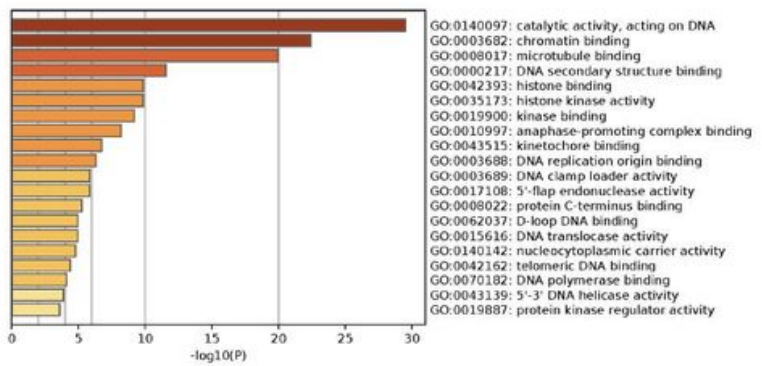

D

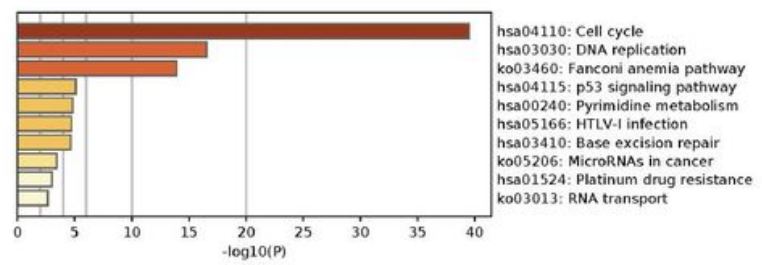

F

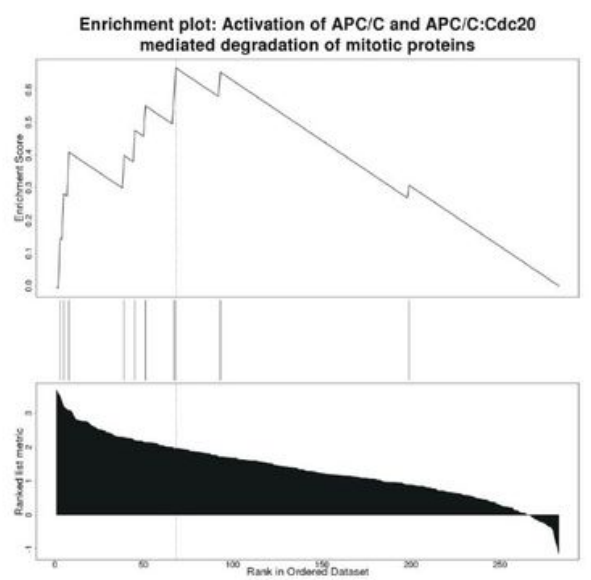

Figure 4

Function and pathway enrichment analysis of CENPF in LUAD. (A-C) Significant Gene Ontology terms of the first 300 genes most closely related to CENPF, containing cell component (CC), molecular function (MF), and biological processes (BP). (D) The important KEGG pathways of the first 300 genes most positively associated with CENPF. (E-F) Significant GSEA results of the first 300 genes most closely related to CENPF, containing KEGG pathways (E) along with Reactome pathways (F). 
A

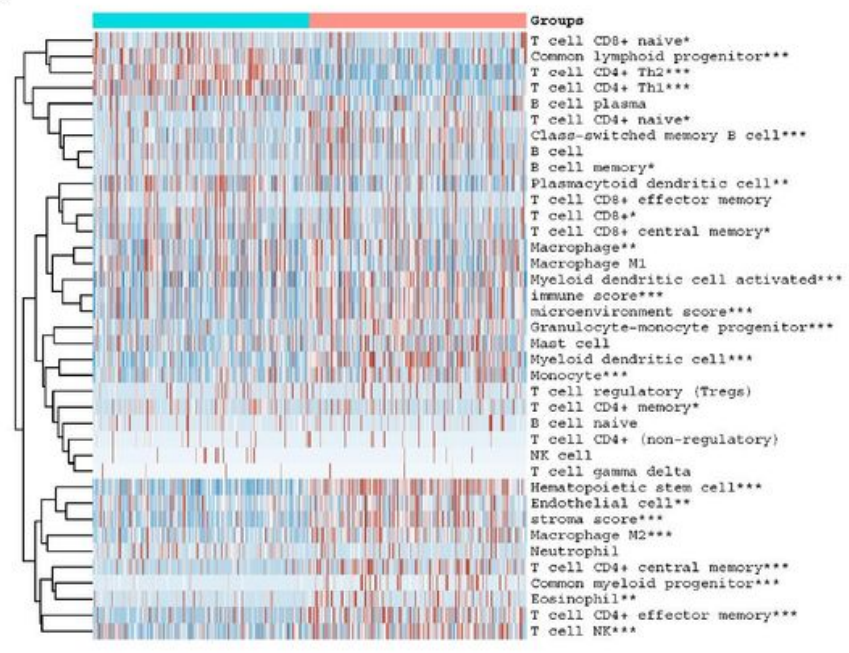

B

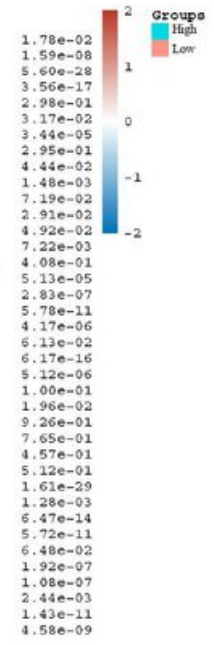

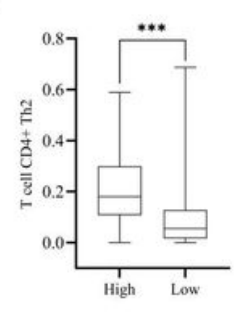

$\mathrm{D}$

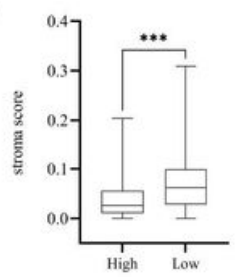

$\mathrm{C}$

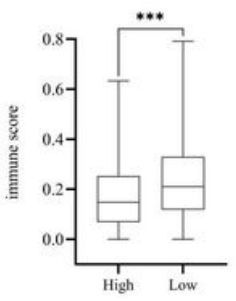

E

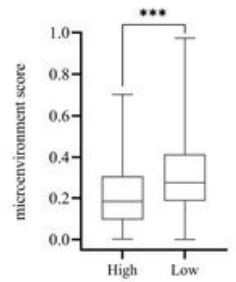

Figure 5

The CENPF expression correlated with immune infiltration. (A) The expression of CENPF remarkably associated with a variety of immune cells infiltration levels according to xCell. (B) T cell CD4+ Th2 infiltration level was substantially higher in LUAD tissues with high CENPF expression than in LUAD tissues with low CENPF expression. (C-E) The microenvironment, stroma and immune scores in high CENPF expression group was evidently lower than the scores in low CENPF expression group. $* p<0: 05$, $* * p<0: 01$, and $* * * p<0: 001$. 
A

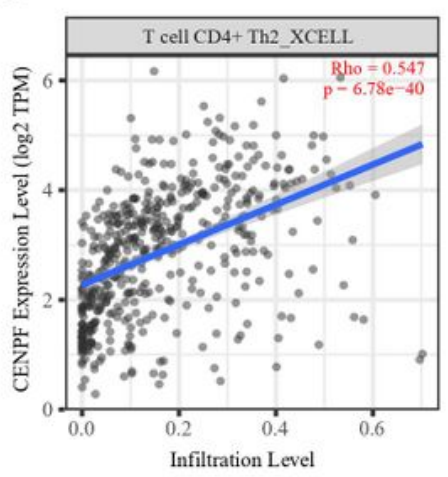

B

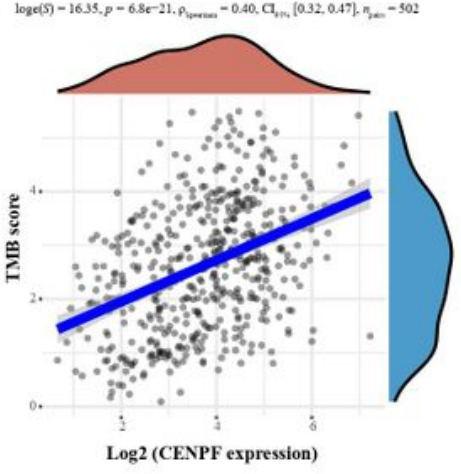

$\mathrm{C}$

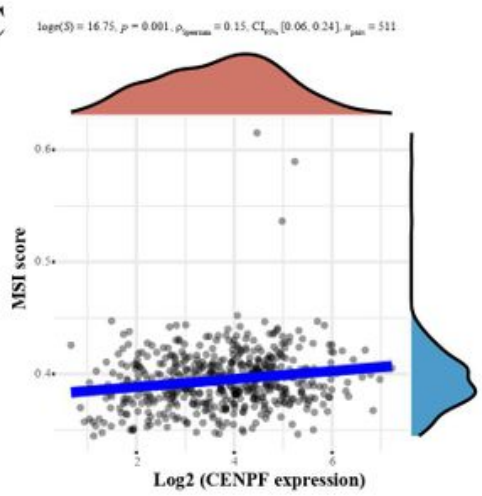

$\mathrm{D}$

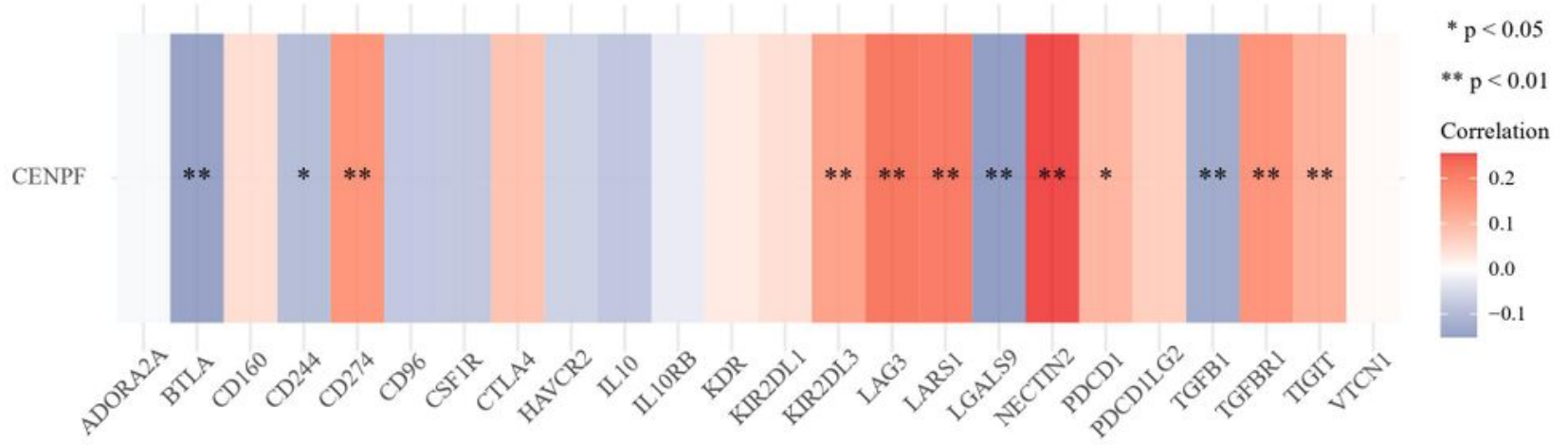

E

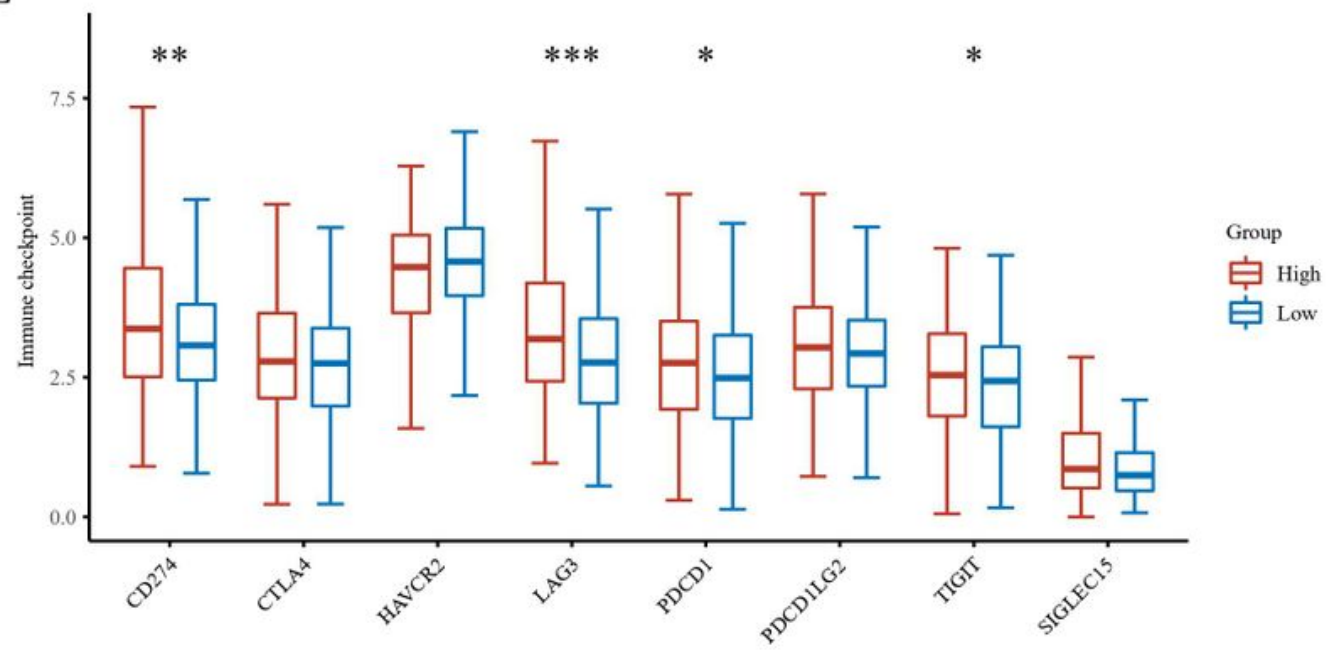

Figure 6

Correlation between TMB/MSI, immune checkpoints and CENPF in LUAD. (A) The relationship between T cell CD4+ Th2 infiltration and the expression of CENPF through Timer2.0 database. (B-C) Correlation analysis in the expression of CENPF and MSI and TMB in LUAD. In the figure, the horizontal axis is the distribution of genes expression, and the vertical axis represents the expression distribution of MSI and TMB scores. (D) CENPF expression was closely associated with the expression of immune checkpoint in 
LUAD, including CD274, KIR2DL3, LAG3, LARS1, NECTIN2, PDCD1, TGFBR1, TIGIT. (E) In the TCGA cohort, the immune checkpoint expression level in group with high CENPF expression was markedly higher in comparison with group with low CENPF expression, containing LAG3, CD274, TIGIT and PDCD1. $* p<$ $0: 05, * * p<0: 01$, and $* * * p<0: 001$.

\section{Supplementary Files}

This is a list of supplementary files associated with this preprint. Click to download.

- SupplementTable1.docx

- SupplementFigure1.pdf

- SupplementFigure2.pdf 